\title{
An Analytical Approach to Study the Blood Flow over a Nonlinear Tapering Stenosed Artery in Flow of Carreau Fluid Model
}

\author{
Riaz Ahmad (D), ${ }^{1}$ Asma Farooqi, ${ }^{1}$ Rashada Farooqi, ${ }^{2}$ Nawaf N. Hamadneh $\left(D,{ }^{3}\right.$ \\ Md Fayz-Al-Asad $\mathbb{D}^{4},{ }^{4}$ Ilyas Khan $\mathbb{D}^{5},{ }^{5}$ Muhammad Sajid $\left(\mathbb{D},{ }^{6}\right.$ Ghulam Bary, ${ }^{1}$ \\ and Muhammad Farooq Saleem Khan ${ }^{7,8}$ \\ ${ }^{1}$ Faculty of Science, Yibin University, Yibin 644000, Sichuan, China \\ ${ }^{2}$ Wah Medical College POF Hospital, Wah Cantt 47040, Pakistan \\ ${ }^{3}$ Department of Basic Sciences, College of Science and Theoretical Studies, Saudi Electronic University, \\ Riyadh 11673, Saudi Arabia \\ ${ }^{4}$ Department of Mathematics, Bangladesh University of Engineering and Technology, Dhaka 1000, Bangladesh \\ ${ }^{5}$ Department of Mathematics, College of Science Al-Zulfi, Majmaah University, Al-Majmaah, Majmaah 11952, Saudi Arabia \\ ${ }^{6}$ Faculty of Materials and Chemical Engineering, Yibin University, Yibin 644000, Sichuan, China \\ ${ }^{7}$ Faculty of International Applied Technology, Yibin University, Yibin 644000, Sichuan, China \\ ${ }^{8}$ School of Environmental Science and Technology, Tsinghua University, Haidian, Beijing, China
}

Correspondence should be addressed to Riaz Ahmad; riazgill2007@gmail.com, Md Fayz-Al-Asad; fayzmath.buet@gmail.com, and Ilyas Khan; i.said@mu.edu.sa

Received 15 March 2021; Accepted 18 July 2021; Published 29 July 2021

Academic Editor: Dan Selisteanu

Copyright (c) 2021 Riaz Ahmad et al. This is an open access article distributed under the Creative Commons Attribution License, which permits unrestricted use, distribution, and reproduction in any medium, provided the original work is properly cited.

\begin{abstract}
The current study provides an analytical approach to analyze the blood flow through a stenosed artery by using the Carreau fluid model. The flow governing equations are derived under the consideration of mild stenosis. Mathematical analysis has been carried out by considering the blood as non-Newtonian nature. Then, the analytical solution has been investigated by using the regular perturbation technique. The solutions obtained by this perturbation are up to the second-order in dimensionless Weissenberg number (We). The performed computations of various parameter values such as velocity, wall shear stress, shear stress, and resistance impedance at the stenotic throat are discussed in detail for different values of Weissenberg number (We). The obtained results demonstrate that for shear-thinning fluid, the fluid velocity increases with the increasing parameter $m$ while opposite behavior is observed with the increase in We. Hence, the presented numerical analysis reveals many aspects of the flow by considering the blood as a non-Newtonian Carreau fluid model, and the presented model can be equally applicable to other biomathematical studies.
\end{abstract}

\section{Introduction}

The study of artery constriction due to the development of stenosis has attained prime importance in fluid dynamics [1-4]. The blood flow in the vessels is a result of the delicate relationship between pressure and area of the fluid. The size of the stenosis determines the flow type. Three types of flow have been studied: mild stenosis as the flow is laminar, moderate stenosis as the flow is a combination of turbulent and laminar, and thirdly the flow depicts turbulent nature when the size of the stenosis is increased. The characteristics of the blood flow depend on the shape and size of the stenosis. Many researchers have analyzed this biomechanical aspect of the flow theoretically and experimentally in recent years. Tang et al. [5] propounded that when the blood pressure is low, stenotic vessels get collapsed and the perfusion of the area beyond stenosis is highly compromised leading to ischemia/infarct. Applications of micropolar fluids in biomedical sciences have received attention for blood flow in arteries [6-8]. 
In the literature, numerous analytical studies related to blood flow through stenosed arteries have been extensively performed [9-13]. In most of these studies, the flow is considered as laminar with mild stenosis [14]. Considerable work has been done for non-Newtonian fluid models. The Carreau fluid model is one of the generalized Newtonian fluid models which is also considered as a viscosity model. This model helps us to explain the behavior of fluid flow in high shear regions and modeling blood at narrow arteries with low shear rates. Firstly, this model was presented by Carreau [15] in his molecular network theories. Then, Siska et al. [16] have proposed a procedure for the terminal velocity of nonspherical particles by using Carreau fluid in transient flow regions. Such regions are responsible for the development of stenosis in the arteries that could lead to stroke, nausea, back pain, etc., specifically with regard to malfunction of the cardiovascular structures. Therefore, the study of blood vessels especially in stenotic arteries has also attained importance in the fluid dynamics field [17].

The shear-thinning and shear-thickening processes of the fluid are well explained by the Carreau fluid model $[18,19]$. This method is a mixture of power-law and Newtonian fluid models. Chhabra and Uhlherr [20] have analyzed the Carreau viscosity equations for shear-thinning elastic liquids. Theoretical analysis of the Carreau fluid model is studied by Bush and Phan-Thein [21]. Later on, Lee [22] discussed the Carreau generalized Newtonian model for error estimations. Tabakova et al. [23] analyzed the flow dynamics in blood vessels by using the Carreau model. They have studied the oscillatory and steady flows and approximated their numerical solutions. Liu and Liu [24] investigated the quantitative analysis of blood flow in tapered stenosed arteries. Their main concern is the heat and mass transfer effect on the fluid. Irfan et al. [25] gave a numerical analysis of the unsteady Carreau fluid model. The flow of blood in the arteries is highly pulsatile with unequal velocity distribution which is the cause of many cardiovascular diseases [26]. Ismail et al. [9] constructed a mathematical model to study the generalized Newtonian blood flow through a tapered artery. Liu et al. [27] considered the effects of tapering and stenosis over the blood flow. Moreover, a numerous range of blood flow phenomena, such as hemodynamics behavior [28], axisymmetric micropolar model [10], heat and mass transfer effects [29], Eyring-Prandtl fluid model [30], and the micropolar fluid model for composite stenosis behavior [31], have been analyzed comprehensively. Flow of bloodbased nanofluids by using the generalized differential quadrature method and other epidemiological models is carried out by many researchers [32-40].

This study aims to investigate the blood flow through a stenosed artery by using the Carreau fluid model. The analytical solution of the governing equations with boundary conditions of the stenotic artery is derived. The perturbation solution is obtained by removing the nonlinearity in the governing equation. Then, the obtained results of the velocity profile, resistance impedance, shear stress, and shear wall stress are shown with rheological parameters of the Carreau fluid model, i.e., We and $m$. Eventually, the graphical results are presented with different values of parameters of interest.

\section{Problem Formulation}

The fundamental equations used in the derivation of the governing equations for the problem considered are

$$
\begin{aligned}
\frac{\partial u}{\partial r}+\frac{u}{r}+\frac{\partial w}{\partial z} & =0 \\
\rho\left(u \frac{\partial}{\partial r}+w \frac{\partial}{\partial z}\right) u & =-\frac{\partial p}{\partial r}+\frac{1}{r} \frac{\partial}{\partial r}\left(r S_{r r}\right)+\frac{\partial}{\partial z}\left(S_{r z}\right)-\frac{S_{\theta \theta}}{r} \\
\rho\left(u \frac{\partial}{\partial r}+w \frac{\partial}{\partial z}\right) w & =-\frac{\partial p}{\partial z}+\frac{1}{r} \frac{\partial}{\partial r}\left(r S_{r z}\right)+\frac{\partial}{\partial z}\left(S_{z z}\right)
\end{aligned}
$$

It is assumed that the properties of blood flowing the cylindrical tube are described by the constitute relation Carreau model given as [41]

$$
\mathbf{S}=\mu\left[1+(\Gamma \dot{\gamma})^{2}\right]^{(m-1) / 2} \dot{\gamma}
$$

Assuming $\Gamma \dot{\gamma} \ll 1$, we can write

$$
\mathbf{S}=\mu\left[1+\left(\frac{m-1}{2}\right)(\Gamma \dot{\gamma})^{2}\right] \dot{\gamma},
$$

where $\Gamma$ is a time constant of the fluid. The geometrical representation of the constricted portion is shown in Figure 1 and defined as

$$
\begin{aligned}
h(z) & =d(z)\left[1-\eta\left(b^{n-1}(z-1)-(z-a)^{n}\right)\right], \quad a \leq z \leq(a+b) \\
& =d(z), \quad \text { otherwise, }
\end{aligned}
$$

with

$$
d(z)=d_{0}+\xi^{*} Z
$$

In the above equation, $\xi^{*}=\tan \phi$ is called the tapering parameter; $a$ is the length of the nonstenotic part, and $b$ is the length of the stenotic section; the radius of the nontapered artery in the nonstenotic section is $d_{0}$; and shape parameter is $n$ which defined the constriction shape, $n=2$ gives the stenosis symmetric behavior. The parameter $\eta$ is written as

$$
\eta=\frac{\delta n^{n /(n-1)}}{d_{0} b^{n}(n-1)},
$$

where $\delta$ is the maximum height of the stenosis defined as

$$
z=a+\frac{b}{\left(1 / n^{(n-1)}\right)} \text {. }
$$

The detailed derivation of the presenting problem is given here

$$
\mathbf{V}=[u(r, z), 0, w(r, z)]
$$




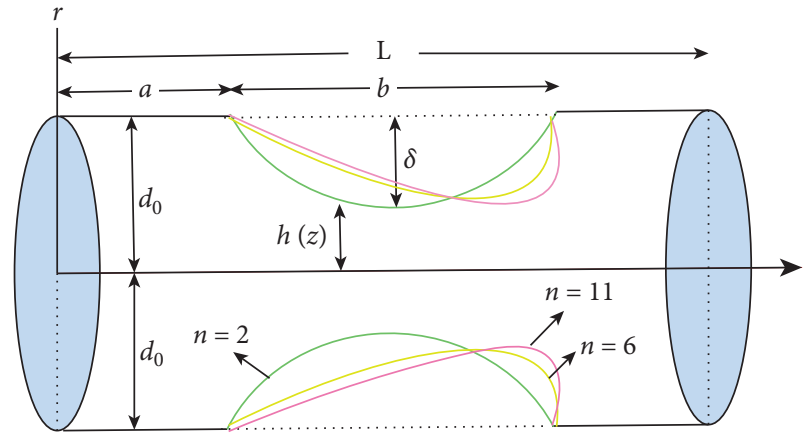

FIGURE 1: Diagram of axially nonsymmetric stenosis in the artery.

$$
\begin{aligned}
& \mathbf{L}=\nabla \mathbf{V}=\left[\begin{array}{lll}
\frac{\partial u}{\partial r} & 0 & \frac{\partial u}{\partial z} \\
0 & \frac{u}{r} & 0 \\
\frac{\partial w}{\partial r} & 0 & \frac{\partial w}{\partial z}
\end{array}\right] \\
& \mathbf{L}^{T}=\left[\begin{array}{lll}
\frac{\partial u}{\partial r} & 0 & \frac{\partial w}{\partial r} \\
0 & \frac{u}{r} & 0 \\
\frac{\partial u}{\partial z} & 0 & \frac{\partial w}{\partial z}
\end{array}\right]
\end{aligned}
$$

Here we defined the rate of strain tensor as given

$$
\begin{gathered}
\dot{\gamma}=\mathbf{L}+\mathbf{L}^{T}=\left[\begin{array}{ccc}
u_{r} & 0 & u_{z} \\
0 & \frac{u}{r} & 0 \\
w_{r} & 0 & w_{z}
\end{array}\right]+\left[\begin{array}{ccc}
u_{r} & 0 & w_{r} \\
0 & \frac{u}{r} & 0 \\
u_{z} & 0 & w_{z}
\end{array}\right], \\
\text { or } \dot{\gamma}=\left[\begin{array}{ccc}
2 u_{r} & 0 & u_{z}+w_{r} \\
0 & 2 \frac{u}{r} & 0 \\
w_{r}+u_{z} & 0 & 2 w_{z}
\end{array}\right],
\end{gathered}
$$

now

$$
\dot{\gamma}=|\dot{\gamma}|=\sqrt{\frac{1}{2} \sum_{i} \sum_{j} \dot{\gamma}_{i j} \dot{\gamma}_{j i}}
$$

Expanding the double sum, we get

$$
\dot{\gamma}=\left[\frac{1}{2}\left(\dot{\gamma}_{11} \dot{\gamma}_{11}+\dot{\gamma}_{12} \dot{\gamma}_{12}+\dot{\gamma}_{13} \dot{\gamma}_{13}+\dot{\gamma}_{21} \dot{\gamma}_{21}+\dot{\gamma}_{22} \dot{\gamma}_{22}+\dot{\gamma}_{23} \dot{\gamma}_{23}+\dot{\gamma}_{31} \dot{\gamma}_{31}+\dot{\gamma}_{32} \dot{\gamma}_{32}+\dot{\gamma}_{33} \dot{\gamma}_{33}\right)\right]^{(1 / 2)}
$$

By choosing the values of the component of $\dot{\gamma}$ from equation (13), we get

$$
\dot{\gamma}=\left[\frac{1}{2} 4 u_{r} u_{r}+\left(u_{z}+w_{r}\right)\left(u_{z}+w_{r}\right)+4 \frac{u}{r} \frac{u}{r}+\left(w_{r}+u_{z}\right)\left(w_{r}+u_{z}\right)+4 w_{z} w_{z}\right]^{(1 / 2)} .
$$

Simplification of the above expression yields

$$
\begin{aligned}
\dot{\gamma} & =\left[\frac{1}{2}\left(4 u_{r}^{2}+2\left(u_{z}+w_{r}\right)^{2}+4 \frac{u^{2}}{r^{2}}+4 w_{z}^{2}\right)\right]^{(1 / 2)}, \\
\text { or } \dot{\gamma} & =\left[2 u_{r}^{2}+\left(u_{z}+w_{r}\right)^{2}+2 \frac{u^{2}}{r^{2}}+2 w_{z}^{2}\right]^{(1 / 2)},
\end{aligned}
$$

by assuming

$$
M=(\Gamma \dot{\gamma})^{2},
$$

we get the following components of the extra stress tensor for the Carreau model:

$$
\left.\begin{array}{l}
S_{r r}=2 \mu\left[1+\left(\frac{m-1}{2}\right) M\right] u_{r} \\
S_{r z}=\mu\left[1+\left(\frac{m-1}{2}\right) M\right]\left(u_{z}+w_{z}\right)=S_{z r}
\end{array}\right\} .
$$


Applying the mild stenotic conditions, we get

$$
\begin{aligned}
& M=\Gamma^{2} w_{r}^{2}, \\
& S_{r r}=0, \\
& S_{\theta \theta}=0, \\
& S_{z z}=0 \\
& S_{r z}=\mu\left[1+\left(\frac{m-1}{2}\right) \Gamma^{2}\left(w_{r}\right)^{2}\right] w_{r}=S_{z r} .
\end{aligned}
$$

Given the above results, the governing equations (1)-(3) can be rewritten as

$$
\begin{aligned}
\frac{\partial p}{\partial r} & =0 \\
\frac{\partial u}{\partial r}+\frac{u}{r}+\frac{\partial w}{\partial z} & =0 \\
\frac{\partial p}{\partial z} & =\frac{\mu}{r} \frac{\partial}{\partial r}\left[r\left[\left[1+\left(\frac{m-1}{2}\right) \Gamma^{2}\left(w_{r}\right)^{2}\right]\left(w_{r}\right)\right]\right] .
\end{aligned}
$$

Dimensionless variables are defined as

$$
\left.\begin{array}{l}
\bar{r}=\frac{r}{d_{0}}, \bar{z}=\frac{z}{b}, \bar{w}=\frac{w}{u_{0}}, \bar{h}=\frac{h}{d_{0}}, \bar{u}=\frac{b u}{u_{0} \delta} \\
\bar{p}=\frac{d_{0}^{m+1} p^{m}}{u_{0}^{m} b \mu} R_{e}=\frac{\rho d_{0}^{m+1}}{u_{0}^{m-2} \mu b}, \bar{S}_{r r}=\frac{b S_{r r}}{u_{0}^{m} \mu} \\
\bar{S}_{r z}=\frac{d_{0}^{m} S_{r z}}{u_{0}^{m} \mu}, \bar{h}=\frac{h}{d_{0}}, \bar{S}_{z z}=\frac{b S_{z z}}{u_{0}^{m} \mu}, \bar{S}_{\theta \theta}=\frac{b S_{\theta \theta}}{u_{0}^{m} \mu}
\end{array}\right\},
$$

and get the following dimensionless governing equation after dropping the bars for simplicity

$$
\begin{array}{r}
\frac{\partial p}{\partial r}=0 \\
\frac{\partial u}{\partial r}+\frac{u}{r}+\frac{\partial w}{\partial z}=0
\end{array}
$$

$$
\frac{\partial p}{\partial z}=\frac{1}{r} \frac{\partial}{\partial r}\left[r\left[\frac{\partial w}{\partial r}+\frac{m-1}{2} \mathrm{We}^{2}\left(\frac{\partial w}{\partial r}\right)^{3}\right]\right],
$$

where $\mathrm{We}=\Gamma u_{0} / d_{0}$ is the dimensionless Weissenberg number.

2.1. Boundary Conditions. The following boundary conditions are applied along with geometrical interpretation:

$$
\begin{aligned}
w=0, & \text { at } r=h(z), \\
\frac{\partial w}{\partial r}=0, & \text { at } r=0,
\end{aligned}
$$

where $h(z)$ is already defined in equation (6).

\section{Employing Perturbation Approach}

To get the perturbation solution for the above defined mathematical model, equation (26) can be rearranged as

$$
\frac{\partial p}{\partial z}=\frac{1}{r} \frac{\partial}{\partial r}\left[r\left[1+\mathrm{We}^{2} \frac{(m-1)}{2}\left(\frac{\partial w}{\partial r}\right)^{2}\right] \frac{\partial w}{\partial r}\right],
$$

and by integrating equation (29), we get

$$
\frac{r^{2}}{2} \frac{\partial p}{\partial z}+C_{1}=\left[r\left[1+\frac{(m-1)}{2} \mathrm{We}^{2}\left(\frac{\partial w}{\partial r}\right)^{2}\right] \frac{\partial w}{\partial r}\right] .
$$

The symmetry condition at the center line $r=0$ yields $C_{1}=0$, and thus we can write equation (30) as

$$
\frac{r}{2} \frac{\partial p}{\partial z}=\left[1+\frac{(m-1)}{2} \mathrm{We}^{2}\left(\frac{\partial w}{\partial r}\right)^{2}\right] \frac{\partial w}{\partial r}
$$

Due to nonlinearity arising in equation (31), we opt perturbation technique to solve it. We assume

$$
\begin{aligned}
& w=w_{0}+\mathrm{We}^{2} w_{1}+\cdots, \\
& p=p_{0}+\mathrm{We}^{2} p_{1}+\cdots, \\
& F=F_{0}+\mathrm{We}^{2} F_{1}+\cdots .
\end{aligned}
$$

Substituting equations (32) and (33) into equations (27) and (34), we find that 


$$
\begin{aligned}
& \frac{r}{2}\left(\frac{\partial p_{0}}{\partial z}+\mathrm{We}^{2} \frac{\partial p_{1}}{\partial z}\right)= \frac{\partial w_{0}}{\partial r}+\mathrm{We}^{2}\left[\frac{\partial w_{1}}{\partial r}+\frac{m-1}{2}\left(\frac{\partial w_{0}}{\partial r}\right)^{3}\right] \\
&+\mathrm{We}^{4}\left[\frac{m-1}{2}\left(\frac{\partial w_{0}}{\partial r}\right)^{2} \frac{\partial w_{1}}{\partial r}+2\left(\frac{m-1}{2}\right) \frac{\partial w_{1}}{\partial r}\left(\frac{\partial w_{0}}{\partial r}\right)^{2}\right] \\
&+\mathrm{We}^{6}\left[\frac{m-1}{2}\left(\frac{\partial w_{1}}{\partial r}\right)^{4} \frac{\partial w_{0}}{\partial r}+2\left(\frac{m-1}{2}\right) \frac{\partial w_{0}}{\partial r}\left(\frac{\partial w_{1}}{\partial r}\right)^{2}\right] \\
&+\mathrm{We}^{8} \frac{m-1}{2}\left(\frac{\partial w_{1}}{\partial r}\right)^{5}, \\
& w_{0}+\mathrm{We}^{2} w_{1}=0, \quad \text { at } r=h(z) .
\end{aligned}
$$

Now comparing the coefficients of various powers of We in equations (35) and (36), the following systems can be obtained.

System of order $\mathrm{We}^{0}$ :

$$
\begin{aligned}
\frac{r}{2} \frac{\partial p_{0}}{\partial z} & =\frac{\partial w_{0}}{\partial r} \\
w_{0} & =0, \quad \text { at } r=h(z) .
\end{aligned}
$$

System of order $\mathrm{We}^{2}$ :

$$
\begin{aligned}
\frac{r}{2} \frac{\partial p_{0}}{\partial z} & =\frac{\partial w_{1}}{\partial r}+\left(\frac{m-1}{2}\right)\left(\frac{\partial w_{0}}{\partial r}\right)^{3}, \\
w_{1} & =0, \quad \text { at } r=h(z) .
\end{aligned}
$$

Solving these system results in the following values of $w_{0}$ and $w_{1}$ :

$$
\begin{aligned}
& w_{0}=\frac{1}{4} \frac{\partial p_{0}}{\partial z}\left[r^{2}-h^{2}\right] \\
& w_{1}=\frac{1}{4} \frac{\partial p_{1}}{\partial z}\left[r^{2}-h^{2}\right]+\frac{(m-1)}{64}\left(\frac{\partial p_{0}}{\partial z}\right)^{3}\left[h^{4}-r^{4}\right] .
\end{aligned}
$$

The definition of flow rate allows us to write

$$
F_{0}=2 \pi \int_{0}^{h} r w_{0} \mathrm{~d} r
$$

inserting the value $w_{0}$ from equation (39) leads to the following expression:

$$
\frac{\partial p_{0}}{\partial z}=-\frac{8}{\pi h^{4}} F_{0},
$$

similarly, we can write

$$
F_{1}=2 \pi \int_{0}^{h} r w_{1} \mathrm{~d} r
$$

and thus,

$$
\frac{\partial p_{1}}{\partial z}=-\frac{8 F_{1}}{h^{4} \pi}-(m-1) \frac{16 \times 8}{3 \pi^{3} h^{10}}\left(F_{0}\right)^{3} .
$$

We summarize the results of the perturbation series through an order $\mathrm{We}^{2}$. By substituting equations (39)-(44), we get

$$
\begin{aligned}
w= & -\frac{2}{\pi h^{4}} F_{0}\left[r^{2}-h^{2}\right]+\frac{\mathrm{We}^{2}}{4}\left[r^{2}-h^{2}\right]\left[-\frac{8 F_{1}}{h^{4} \pi}-(m-1) \frac{128}{3 \pi^{3} h^{10}}\left(F_{0}\right)^{3}\right] \\
& -\frac{8 \mathrm{We}}{\pi^{3} h^{12}}(m-1)\left[h^{4}-r^{4}\right]\left(F_{0}\right)^{3}, \\
\frac{\partial p}{\partial z}= & -\frac{8}{\pi h^{4}} F_{0}-\mathrm{We}^{2}\left[\frac{8 F_{1}}{h^{4} \pi}+(m-1) \frac{16 \times 8}{3 \pi^{3} h^{10}}\left(F_{0}\right)^{3}\right] .
\end{aligned}
$$




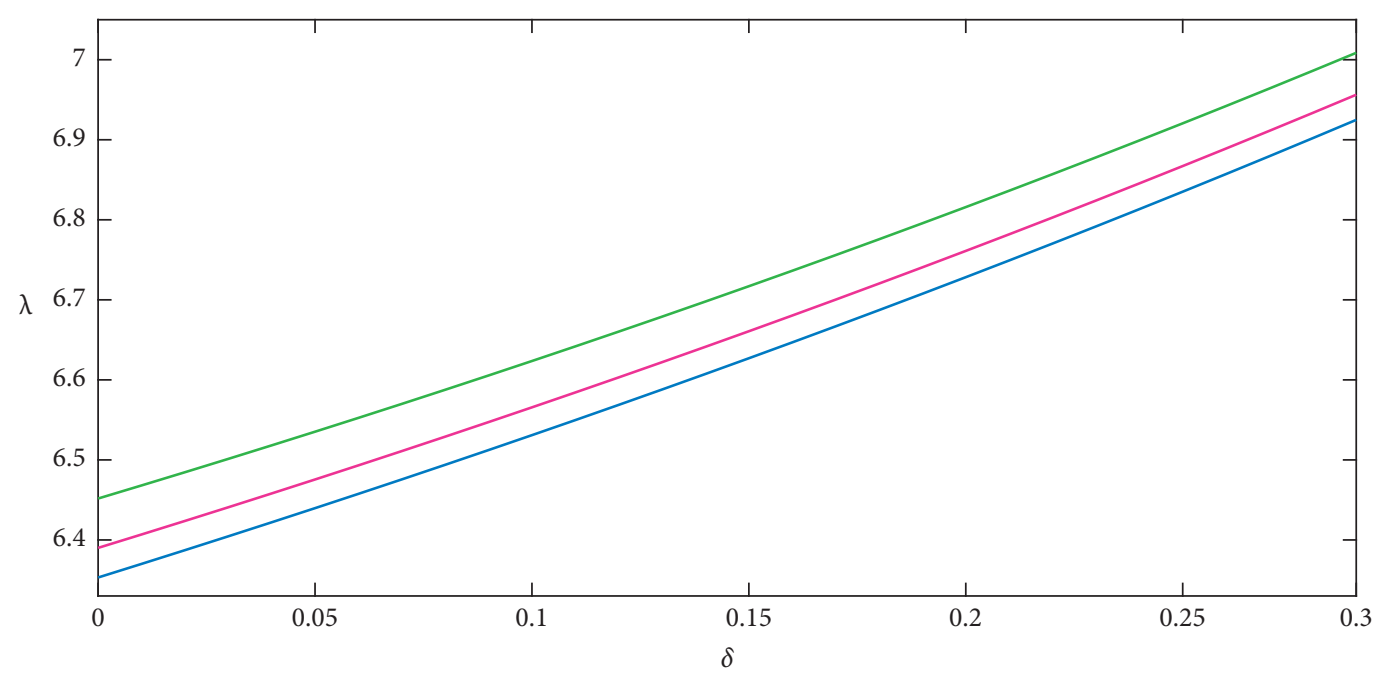

$\begin{aligned} \mathrm{We} & =0.1 \\ \mathrm{We} & =0.2 \\ \mathrm{We} & =0.3\end{aligned}$

Figure 2: Variation of resistance for the Carreau model for $m=2, n=2, L=1$, and $F=0.6$.

If we define

$$
\begin{aligned}
F^{(2)} & =F_{0}+\mathrm{We}^{2} F_{1}, \\
F_{0} & =F^{(2)}-\mathrm{We}^{2} F_{1} .
\end{aligned}
$$

On substituting these expressions into equations (45) and (46) and retaining only terms up to $\mathrm{O}\left(\mathrm{We}^{2}\right)$, we obtain

$$
\begin{aligned}
& \frac{\partial p}{\partial z}=-\frac{8 F}{\pi h^{4}}-\mathrm{We}^{2}(m-1) \frac{16 \times 8}{3 \pi^{3} h^{10}} F^{3}, \\
& w=-\frac{2 F}{\pi h^{4}}\left[r^{2}-h^{2}\right]-\frac{32 \mathrm{We}^{2}}{3 \pi^{3} h^{10}} F^{3}\left[r^{2}-h^{2}\right](m-1)-\frac{8 \mathrm{We}}{\pi^{3} h^{12}} F^{3}(m-1)\left[h^{4}-r^{4}\right] . \\
& \qquad \Delta p=\int_{0}^{(L / b)}\left(\frac{8 F}{\pi h^{4}}+\mathrm{We}^{2}(m-1) \frac{128}{3 \pi^{3} h^{10}} F^{3}\right) \mathrm{d} z,
\end{aligned}
$$

$$
\Delta p=\int_{0}^{(L / b)}\left(-\frac{\mathrm{d} p}{\mathrm{~d} z}\right) \mathrm{d} z
$$

and similarly, the resistance impedance is defined by the given expression:

and on substituting the value of $(\mathrm{d} p / \mathrm{d} z)$ from equation (48), we derive

$$
\lambda=\frac{\Delta p}{F}=\left.\int_{0}^{(a / b)} R(z)\right|_{h=1+\xi z} \mathrm{~d} z+\int_{(a / b)}^{(a / b)+1} R(z) \mathrm{d} z+\left.\int_{(a / b)+1}^{(L / b)} R(z)\right|_{h=1+\xi z} \mathrm{~d} z,
$$

and the nondimensional shear stress for the Carreau law since model is given as

$$
S_{r z}=\frac{\partial w}{\partial r}+\mathrm{We}^{2}\left(\frac{m-1}{2}\right)\left(\frac{\partial w}{\partial r}\right)^{3}
$$

$$
w=-\frac{2 F}{\pi h^{4}}\left[r^{2}-h^{2}\right]-\frac{32 \mathrm{We}}{3 \pi^{3} h^{10}} F^{3}\left[r^{2}-h^{2}\right](m-1)-\frac{8 \mathrm{We}}{\pi^{3} h^{12}} F^{3}(m-1)\left[h^{4}-r^{4}\right]
$$




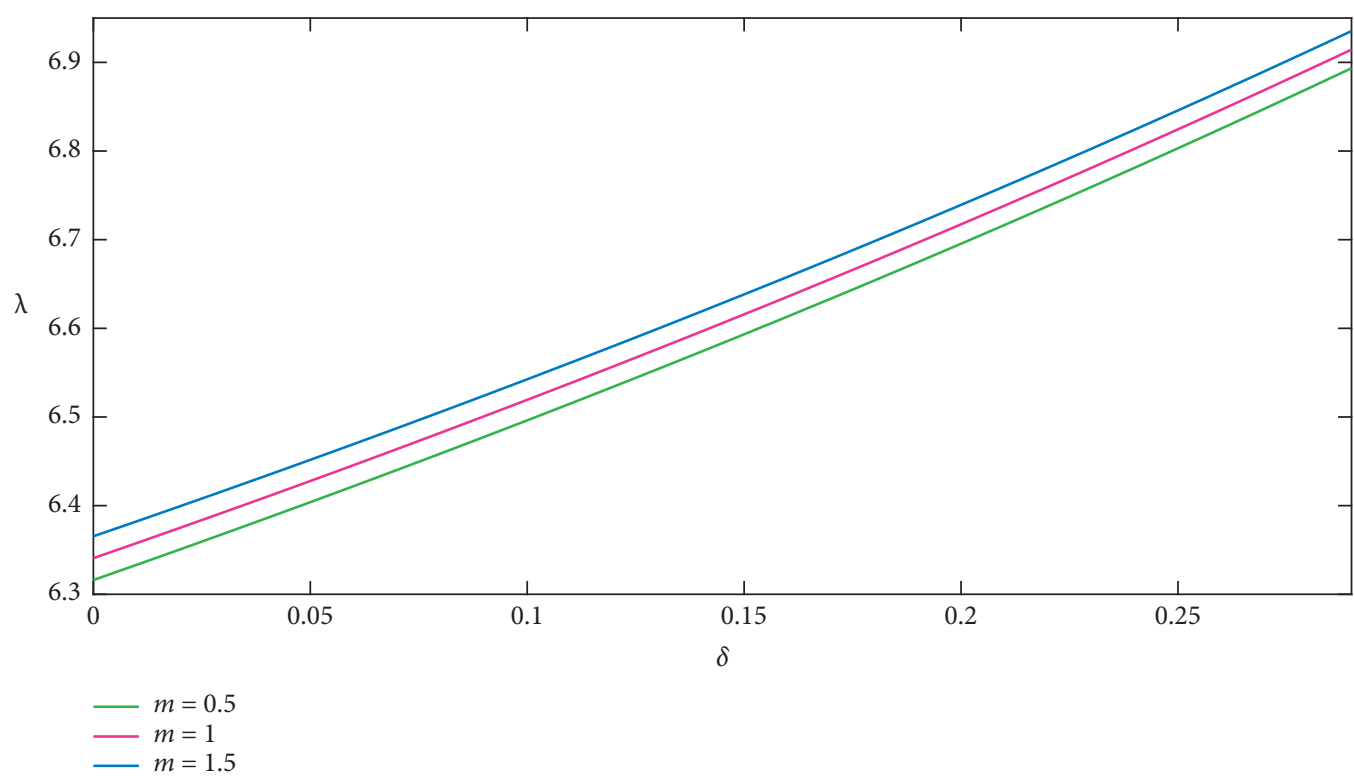

Figure 3: Variation of resistance for the Carreau model for $\mathrm{We}=0.2, n=2, L=1$, and $F=0.6$.

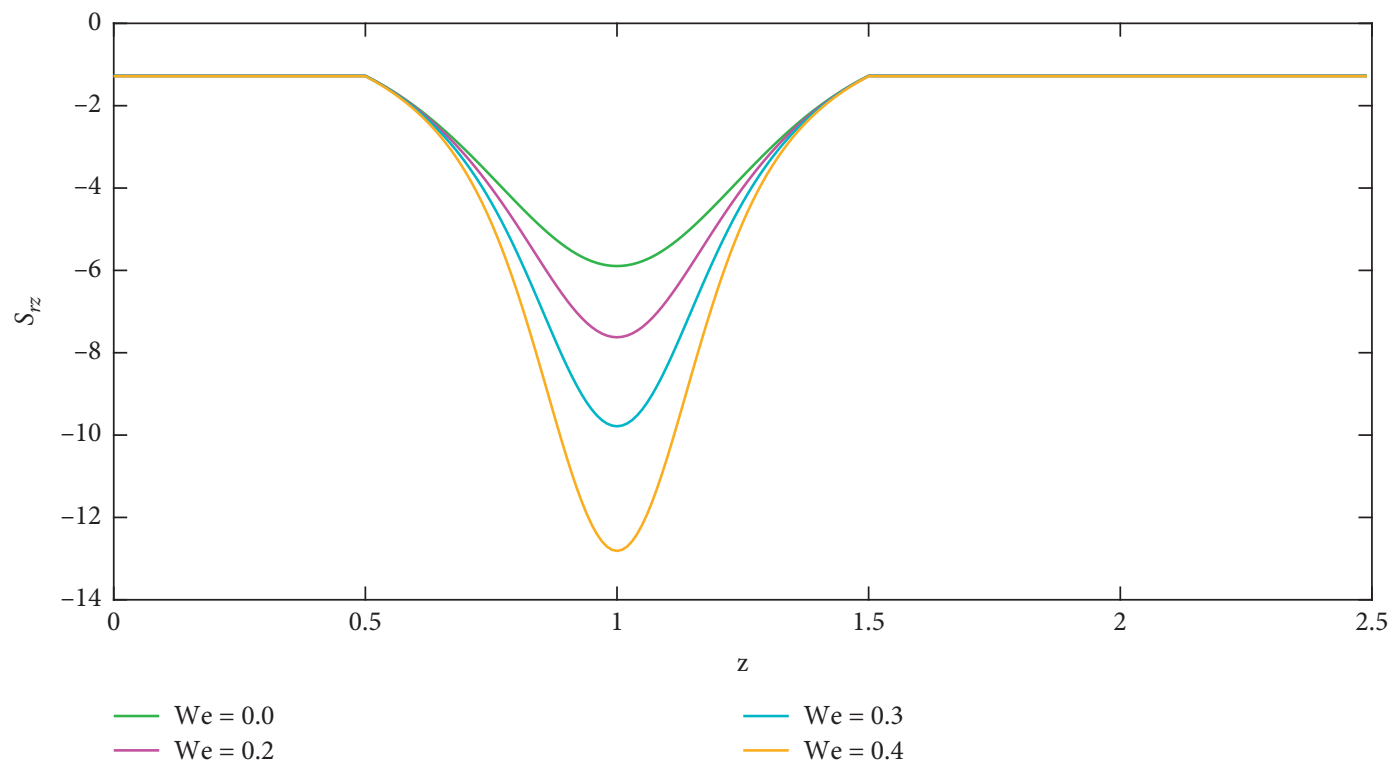

FIgURE 4: Wall shear stress variation for the Carreau model for $n=2, L=1, F=1$, and $m=0.8$.

we obtain and thus equation (53) gives

$$
\frac{\partial w}{\partial r}=-\frac{4 F r}{\pi h^{4}}-\frac{64 \mathrm{We}^{2} F^{3}(m-1) r}{3 \pi^{3} h^{10}}+\frac{32 r^{3} \mathrm{We}^{2}}{\pi^{3} h^{12}} F^{3}(m-1),
$$

$$
\begin{aligned}
S_{r z}= & -\frac{4 F r}{\pi h^{4}}-\frac{64 \mathrm{We}^{2} F^{3}(m-1) r}{3 \pi^{3} h^{10}}+\frac{32 r^{3} \mathrm{We}^{2}}{\pi^{3} h^{12}} F^{3}(m-1) \\
& +\mathrm{We}^{2}\left(\frac{m-1}{2}\right)\left(-\frac{4 F r}{\pi h^{4}}-\frac{64 \mathrm{We}^{2} F^{3}(m-1) r}{3 \pi^{3} h^{10}}+\frac{32 r^{3} \mathrm{We}^{2}}{\pi^{3} h^{12}} F^{3}(m-1)\right)^{3} .
\end{aligned}
$$




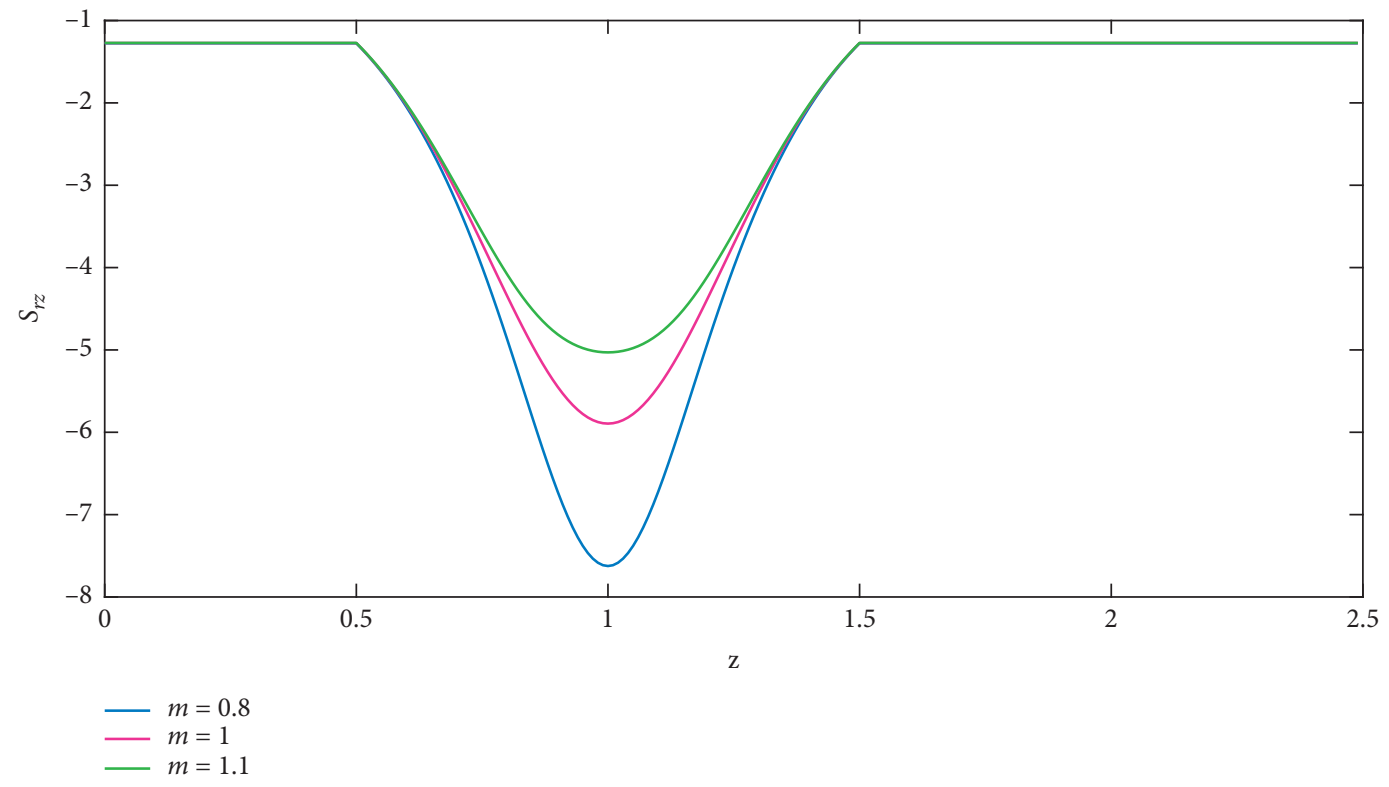

Figure 5: Wall shear stress variation for the Carreau model for $n=2, L=1, F=1$, and We $=0.2$.

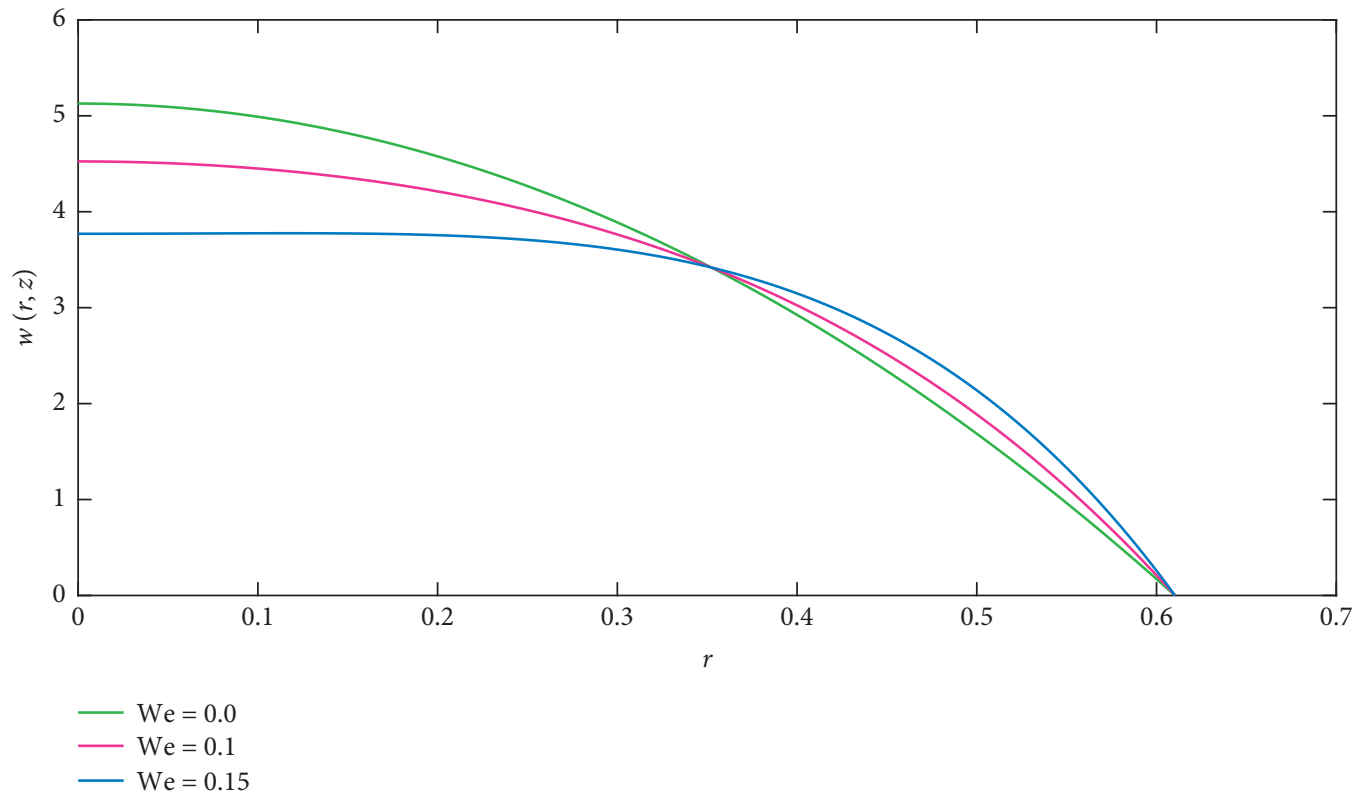

Figure 6: Velocity variation for the Carreau model for $n=2, L=1, F=3$, and $m=0.5$.

Finally, at maximum height, i.e., $h=1-\delta$, the wall shear stress of the stenosis can be written as

$$
\tau_{s}=\left.S_{r z}\right|_{h=1-\delta}=\frac{4}{\pi(1-\delta)^{3}}\left[-F-\frac{16 \mathrm{We}}{3 \pi^{2}(1-\delta)^{6}}(m-1) F^{3}+\frac{8 \mathrm{We}^{2}(m-1)}{\pi^{2}(1-\delta)^{8}} F^{3}\right] .
$$




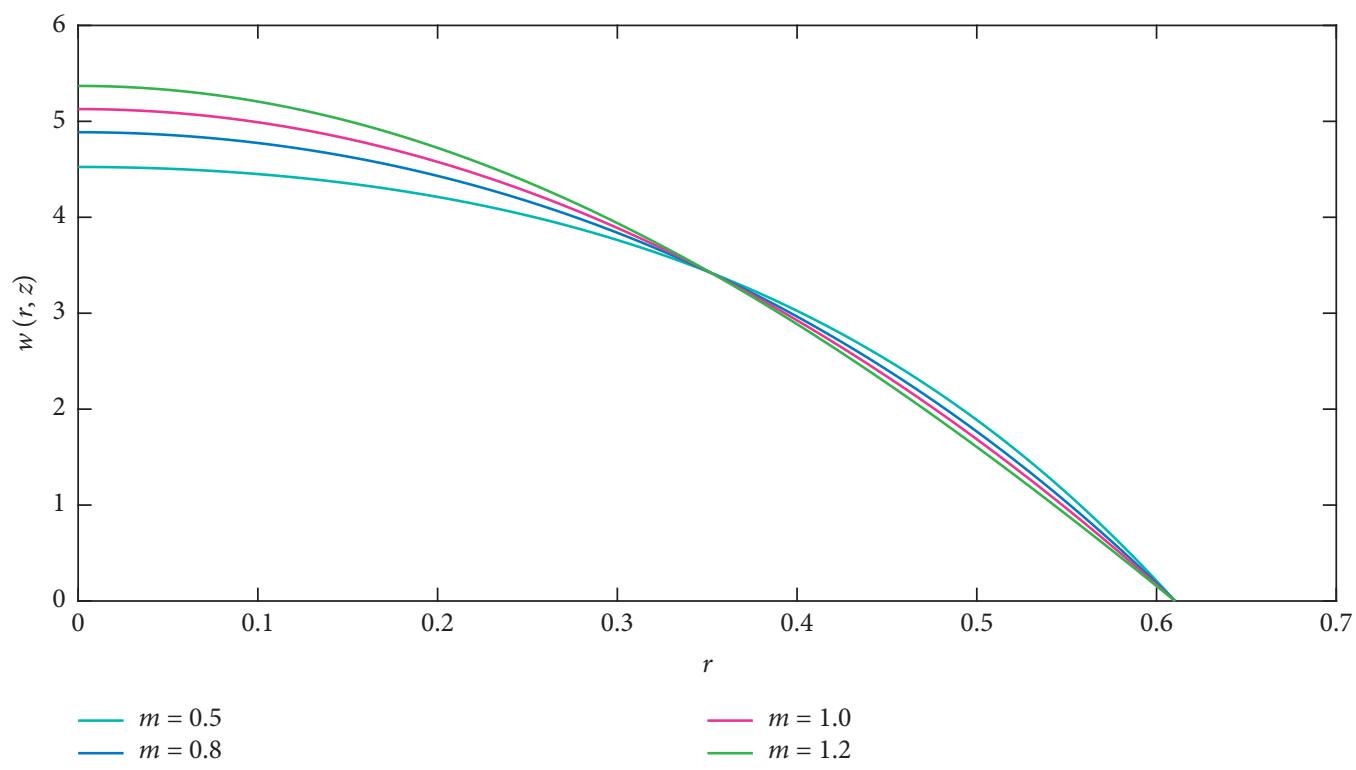

Figure 7: Velocity variation for the Carreau model for $n=3, L=1, F=3$, and $\mathrm{We}=0.1$.

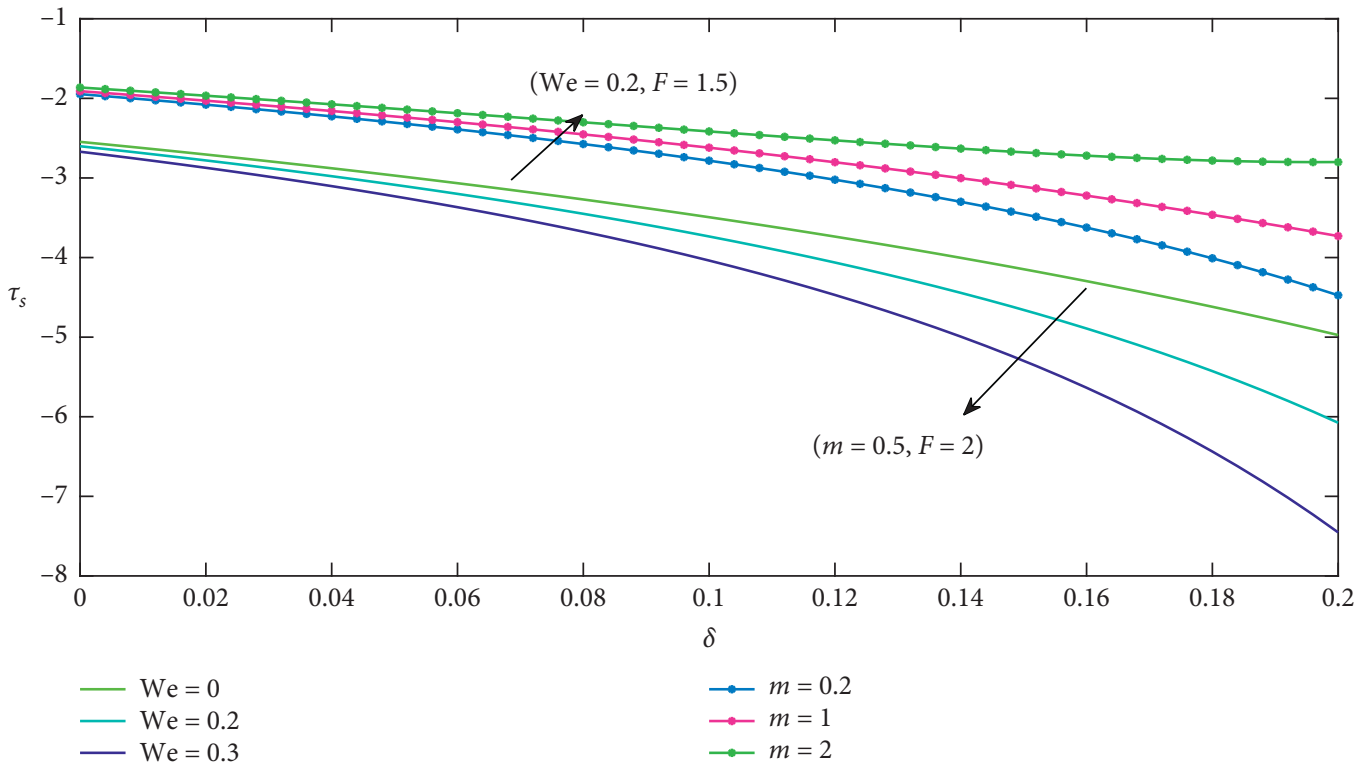

FIGURE 8: Variation of shear stress at the stenotic throat.

\section{Graphical Results and Discussion}

In this section, the effect of rheological parameters of the Carreau fluid model, i.e., We and $m$ on velocity profile, resistance impedance, wall shear stress, and shear stress at the stenotic throat, is graphically performed and discussed. The Weissenberg number is the ratio of the relaxation time of the fluid and a specific process time. Relaxation time increases when we increase the Weissenberg number and velocity field easily increases and skin friction decreases. Another aspect of increasing the Weissenberg number is that it reduces the magnitude of the fluid velocity for shear-thinning fluid, while it arises for the shear-thickening fluid. Figure 2 shows that resistance impedance is an increasing function of the Weissenberg number We.

In Figure 3, it is observed that the resistance impedance increases as there is an increase in power-law index $m$. It explains the the fluid shear-thinning behavior $(m<1)$ slowly compared to Newtonian $(m=1)$ and shear-thickening $(m>1)$ fluids.

The behavior of shear stress for We and $m$ is shown in Figures 4 and 5. It is observed that the magnitude of wall shear stress increases by increasing We as shown in Figure 4 . The opposite behavior is observed when $m$ is increased as seen in Figure 5. 
The effect of the severity of stenosis on the velocity profile $w$ is shown in Figures 6 and 7. In Figure 6, the velocity profile increases at the center of the channel by increasing $m$.

And in Figure 7, an increase in We decreases the velocity at the channel center. It can be seen that for a fixed value of prescribed flux $F$, the velocity profile $w$ increases near the center, while it decreases near the wall with an increase in the severity of stenosis.

In Figure 8, we noticed that the shear stress at the maximum height of stenosis behaves differently by increasing We and $m$. It is analyzed that shear stress by fixing Weissenberg number We and flow rate $F$ at the stenosis throat increases with an increase in $m$. While shear stress decreases with an increase in We with defined values of power-law index $m$ and flow rate $F$.

\section{Conclusion}

In this study, we have analyzed the numerical solution of the blood flow with its non-Newtonian nature. Analytical solutions are derived from the given governing equations. The presented computation results of various parameter values, namely, velocity, wall shear stress, shear stress, and resistance impedance at the stenotic throat, are studied in detail for different values of Weissenberg number (We) and power-law index $m$. From the analysis of the related facts and figures, the following results are concluded:

(1) The velocity profile increases at the center of the channel by increasing $m$. However, an increase in We decreases the velocity at the channel center.

(2) Resistance impedance is an increasing function of We and $m$.

(3) The magnitude of shear stress increases by increasing $\mathrm{We}$, while it shows the opposite behavior when $m$ is increased.

(4) Shear stress at the maximum height of stenosis behaves differently by increasing We and $m$.

Conclusively, the present work may be an improvement in the analysis of pulsatile blood flow through a mild stenotic tapering artery.

\section{Nomenclature}

We: Weissenberg number

$\Gamma: \quad$ Time constant of the fluid

$\xi^{*}$ : Tapering parameter

$a$ : Length of the nonstenotic part

$b$ : $\quad$ Length of the stenotic section

$d_{0}$ : Radius of the nontapered artery in the nonstenotic section

$n: \quad$ Shape parameter

$\delta: \quad$ Maximum height of the stenosis

$\tau_{s}: \quad$ Wall shear stress

$\Delta p:$ Pressure drop

$\lambda: \quad$ Resistance impedance

$m$ : Power-law index

$w$ : Velocity profile
F: $\quad$ Flow rate

$S_{r z}:$ Shear stress

S: $\quad$ Extra stress tensor

$\theta$ : Tapering angle.

\section{Data Availability}

No data were required to perform this research.

\section{Conflicts of Interest}

The authors declare that there are no conflicts of interest.

\section{References}

[1] R. Nasrin, A. Hossain, and I. Zahan, "Blood flow analysis inside a stenotic artery using power-law fluid model," Research and Development in Material Science, vol. 13, no. 1, pp. 1360-1368, 2020.

[2] R. Ahmad, A. Farooqi, J. Zhang, and N. Ali, "Steady flow of a power law fluid through a tapered non-symmetric stenotic tube," Applied Mathematics and Nonlinear Sciences, vol. 4, no. 1, pp. 249-260, 2019.

[3] Y. Pratumwal, W. Limtrakarn, S. Muengtaweepongsa et al., "Whole blood viscosity modeling using power law, Casson, and Carreau Yasuda models integrated with image scanning U-tube viscometer technique," Songklanakarin Journal of Science and Technology, vol. 39, no. 5, pp. 625-631, 2018.

[4] M. Y. A. Jamalabadi, M. Daqiqshirazi, H. Nasiri, M. R. Safaei, and T. K. Nguyen, "Modeling and analysis of biomagnetic blood Carreau fluid flow through a stenosis artery with magnetic heat transfer: a transient study," PLoS One, vol. 13, no. 2, pp. 1-32, 2018.

[5] D. Tang, C. Yang, S. Kobayashic, and D. N. Ku, "Generalized finite difference method for 3-D viscous flow in stenotic tubes with large wall deformation and collapse," Applied Numerical Mathematics, vol. 38, no. 2, pp. 49-68, 2001.

[6] T. Elnaqeeb, "Modeling of Au(NPs)-blood flow through a catheterized multiple stenosed artery under radial magnetic field," The European Physical Journal Special Topics, vol. 228, no. 12, pp. 2695-2712, 2019.

[7] K. S. Mekheimer, T. Elnaqeeb, M. A. El Kot, and F. Alghamdi, "Simultaneous effect of magnetic field and metallic nanoparticles on a micropolar fluid through an overlapping stenotic artery: blood flow model," Physics Essays, vol. 29, no. 2, pp. 272-283, 2016.

[8] T. Elnaqeeb, N. A. Shah, and K. S. Mekheimer, "Hemodynamic characteristics of gold nanoparticle blood flow through a tapered stenosed vessel with variable nanofluid viscosity," BioNanoScience, vol. 9, no. 2, pp. 245-255, 2019.

[9] Z. Ismail, I. Abdullah, N. Mustapha, and N. Amin, "A powerlaw model of blood flow through a tapered overlapping stenosed artery," Applied Mathematics and Computation, vol. 195, no. 2, pp. 669-680, 2008.

[10] K. S. Mekheimer and M. A. E. Kot, "The micropolar fluid model for blood flow through a tapered artery with a stenosis," Acta Mechanica Sinica, vol. 24, no. 6, pp. 637-644, 2008.

[11] I. Abdullah and N. Amin, "A micropolar fluid model of blood flow through a tapered artery with a stenosis," Mathematical Methods in the Applied Sciences, vol. 33, no. 16, pp. 1910-1923, 2010.

[12] K. S. Mekheimer and M. A. El Kot, "Mathematical modelling of unsteady flow of a Sisko fluid through an anisotropically 
tapered elastic arteries with time-variant overlapping stenosis," Applied Mathematical Modelling, vol. 36, no. 11, pp. 5393-5407, 2012.

[13] D. S. Sankar and U. Lee, "Mathematical modeling of pulsatile flow of non-Newtonian fluid in stenosed arteries," Communications in Nonlinear Science and Numerical Simulation, vol. 14, no. 7, pp. 2971-2981, 2009.

[14] K. W. Lee and X. Y. Xu, "Modelling of flow and wall behaviour in a mildly stenosed tube," Medical Engineering \& Physics, vol. 24, no. 9, pp. 575-586, 2002.

[15] P. J. Carreau, "Rheological equations from molecular network theories," Transactions of the Society of Rheology, vol. 16, no. 1, pp. 99-127, 1972.

[16] B. Šiška, H. Bendova, and I. MacHac, "Terminal velocity of non-spherical particles falling through a Carreau model fluid," Chemical Engineering and Processing, vol. 44, no. 12, pp. 1312-1319, 2005.

[17] N. Kutev, S. Tabakova, and S. Radev, "Approximation of the oscillatory blood flow using the Carreau viscosity model," in Proceedings of the International Conference on Mechanics-Seventh Polyakhov's Reading, pp. 1-4, Saint Petersburg, Russia, Feburary 2015.

[18] A. G. Usman and S. Kausar, "Numerical solution of the partial differential equations that model the steady three-dimensional flow and heat transfer of Carreau fluid between two stretchable rotatory disks," Numerical Methods for Partial Differential Equations, pp. 1-29, 2020.

[19] M. I. Khan, S. Qayyum, M. Nigar, Y. Chu, and S. Kadry, "Dynamics of Arrhenius activation energy in flow of Carreau fluid subject to Brownian motion diffusion," Numerical Methods for Partial Differential Equations, pp. 1-21, 2020.

[20] R. P. Chhabra and P. H. T. Uhlherr, "Creeping motion of spheres through shear-thinning elastic fluids described by the Carreau viscosity equation," Rheologica Acta, vol. 19, no. 2, pp. 187-195, 1980.

[21] M. B. Bush and N. Phan-Thien, "Drag force on a sphere in creeping motion throug a carreau model fluid," Journal of Nonnewtonian Fluid Mechanics, vol. 16, no. 3, pp. 303-313, 1984.

[22] H. C. Lee, "An adaptively refined least-squares finite element method for generalized Newtonian fluid flows using the Carreau model," SIAM Journal on Scientific Computing, vol. 36, no. 1, pp. 193-218, 2014.

[23] S. Tabakova, E. Nikolova, and S. Radev, "Carreau model for oscillatory blood flow in a tube," American Institute of Physics, vol. 336, pp. 336-343, 2014.

[24] Y. Liu and W. Liu, "Blood flow analysis in tapered stenosed arteries with the influence of heat and mass transfer," Journal of Applied Mathematics and Computing, vol. 63, no. 1-2, pp. 523-541, 2020.

[25] M. Irfan, K. Rafiq, W. A. Khan, and M. Khan, "Numerical analysis of unsteady Carreau nanofluid flow with variable conductivity," Applied Nanoscience, vol. 10, no. 8, pp. 3075-3084, 2020.

[26] D. S. Sankar and K. Hemalatha, "Pulsatile flow of HerschelBulkley fluid through stenosed arteries-a mathematical model," International Journal of Non-Linear Mechanics, vol. 41, no. 8, pp. 979-990, 2006.

[27] G. T. Liu, X. J. Wang, B. Q. Ai, and L. G. Liu, "Numerical study of pulsating flow through a tapered artery with stenosis," Chinese Journal of Physics, vol. 42, no. 4, pp. 401-409, 2004.

[28] J. Hun, C. J. Wook, and P. C. Guk, "Asymmetric flows of nonNewtonian fluids in symmetric stenosed artery," KoreaAustralia Rheology Journal, vol. 16, no. 2, pp. 101-108, 2004.
[29] N. S. Akbar, "Heat and mass transfer effects on Carreau fluid model for blood flow through a tapered artery with a stenosis," International Journal of Biomathematics, vol. 7, no. 1, pp. 1-21, 2014.

[30] N. S. Akbar, "Eyring Prandtl fluid flow with convective boundary conditions in small intestines," International Journal of Biomathematics, vol. 6, no. 5, pp. 1-13, 2013.

[31] R. Ellahi, S. U. Rahman, M. M. Mudassar Gulzar, S. Nadeem, and K. Vafai, "A mathematical study of non-Newtonian micropolar fluid in arterial blood flow through composite stenosis," Applied Mathematics \& Information Sciences, vol. 8, no. 4, pp. 1567-1573, 2014.

[32] A. Wakif, "A novel numerical procedure for simulating steady MHD convective flows of radiative casson fluids over a horizontal stretching sheet with irregular geometry under the combined influence of temperature-dependent viscosity and thermal conductivity," Mathematical Problems in Engineering, vol. 2020, Article ID 1675350, 20 pages, 2020.

[33] M. Qasim, Z. Ali, A. Wakif, and Z. Boulahia, "Numerical simulation of MHD peristaltic flow with variable electrical conductivity and Joule dissipation using generalized differential quadrature method," Communications in Theoretical Physics, vol. 71, no. 509, pp. 509-518, 2019.

[34] M. Ashraf, M. Qasim, A. Wakif, M. Afridi, and I. Animasaun, "A generalized differential quadrature algorithm for simulating magnetohydrodynamic peristaltic flow of blood-based nanofluid containing magnetite nanoparticles: a physiological application," Numerical Methods for Partial Differential Equations, vol. 20, pp. 1-27, 2020.

[35] R. Ahmad, A. Farooqi, J. Zhang, I. Khan, and E.-S. M. Sherif, "Analysis of transport and mixing phenomenon to invariant manifolds using LCS and KAM theory approach in unsteady dynamical systems," IEEE Access, vol. 8, pp. 141057-141065, 2020.

[36] A. Farooqi, R. Ahmad, H. Alotaibi, T. Nofal, R. Farooqi, and I. Khan, "A comparative epidemiological stability analysis of predictor corrector type non-standard finite difference scheme for the transmissibility of measles," Results in Physics, vol. 21, Article ID 103756, 2021.

[37] A. Farooqi, R. Ahmad, R. Farooqi et al., "An accurate predictor-corrector-type nonstandard finite difference scheme for an SEIR epidemic model," Journal of Mathematics, vol. 2020, Article ID 8830829, 18 pages, 2020.

[38] G. Bary, W. Zhang, P. Ru, and J. Yang, "Analyses of multipion Bose-Einstein correlations for granular sources with coherent pion-emission droplets," Chinese Physics C, vol. 21, Article ID 024106, 2021.

[39] G. Bary, P. Ru, and W. Zhang, "Analyses of multi-pion Hanbury Brown-Twiss correlations for the pion-emitting sources with Bose-Einstein condensation," Journal of Physics G: Nuclear and Particle Physics, vol. 45, Article ID 065102, 2018.

[40] G. Bary, P. Ru, and W. Zhang, "Normalized multi-pion Hanbury-Brown- Twiss correlation functions of pion-emitting sources with Bose-Einstein condensation," Journal of Physics G: Nuclear and Particle Physics, vol. 46, Article ID 115107, 2019.

[41] A. El Hakeem Abd El Naby, A. E. M. Abd El Kareem, and M. F. Kareem, "Separation in the flow through peristaltic motion of a Carreau fluid in uniform tube," Physica A: Statistical Mechanics and Its Applications, vol. 343, pp. 1-14, 2004. 